\title{
Reading Documents for Bayesian Online Change Point Detection
}

\author{
Taehoon Kim and Jaesik Choi \\ School of Electrical and Computer Engineering \\ Ulsan National Institute of Science and Technology \\ Ulsan, Korea \\ \{carpedm20,jaesik\}@unist.ac.kr
}

\begin{abstract}
Modeling non-stationary time-series data for making predictions is a challenging but important task. One of the key issues is to identify long-term changes accurately in time-varying data. Bayesian Online Change Point Detection (BO-CPD) algorithms efficiently detect long-term changes without assuming the Markov property which is vulnerable to local signal noise. We propose a Document based BO-CPD (DBO-CPD) model which automatically detects long-term temporal changes of continuous variables based on a novel dynamic Bayesian analysis which combines a non-parametric regression, the Gaussian Process (GP), with generative models of texts such as news articles and posts on social networks. Since texts often include important clues of signal changes, DBO-CPD enables the accurate prediction of long-term changes accurately. We show that our algorithm outperforms existing BO-CPDs in two real-world datasets: stock prices and movie revenues.
\end{abstract}

\section{Introduction}

Time series data depends on the latent dependence structure which changes over time. Thus, stationary parametric models are not appropriate to represent such dynamic non-stationary processes. Change point analysis (Smith, 1975; Stephens, 1994; Chib, 1998; Barry and Hartigan, 1993) focuses on formal frameworks to determine whether a change has taken place without assuming the Markov property which is vulnerable to local signal noise. When change points are identified, each part of the time series is approximated by specified parametric models under the stationary assumptions. Such change point detection models have successfully been applied to a variety of data, such as stock markets (Chen and Gupta, 1997; Hsu, 1977; Koop and Potter, 2007), analyzing bees' behavior (Xuan and Murphy, 2007), forecasting climates (Chu and Zhao, 2004; Zhao and Chu, 2010), and physics experiments (von Toussaint, 2011). However, offline-based change point analysis suffers from slow retrospective inference which prevents real-time analysis.

Bayesian Online Change Point Detection (BOCPD) (Adams and MacKay, 2007; Steyvers and Brown, 2005; Osborne, 2010; Gu et al., 2013) overcomes this restriction by exploiting efficient online inference algorithms. BO-CPD algorithms efficiently detect long-term changes by analyzing continuous target values with the Gaussian Process (GP), a non-parametric regression method. The GP-based CPD model is simple and flexible. However, it is not straightforward to utilize rich external data such as texts in news articles and posts in social networks.

In this paper, we propose a novel BO-CPD model that improves the detection of change points in continuous signals by incorporating the rich external information implicitly written in texts on top of the long-term change analysis of the GP. In particular, our model finds causes of signal changes in news articles which are influential sources of markets of interests.

Given a set of news articles extracted from the Google News service and a sequence of target, continuous values, our new model, Documentbased Bayesian Online Change Point Detection (DBO-CPD), learns a generative model which represents the probability of a news article given the run length (a length of consecutive observations without a change). By using the new prior, DBOCPD models a dynamic hazard rate $(h)$ which determines the rate at which change points occur.

In the literature, important information is extracted from news articles (Nothman et al., 2012; 


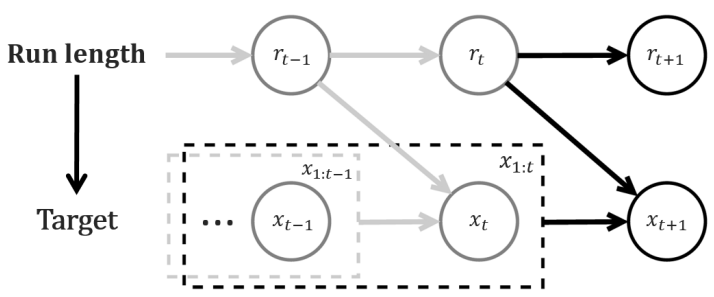

(a) $\mathrm{BO}-\mathrm{CPD}$

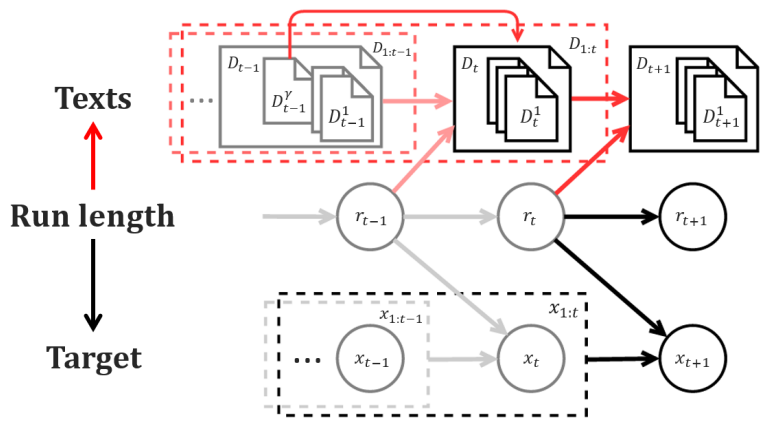

(b) DBO-CPD (this work)

Figure 1: This figures illustrates a graphical representation of BO-CPD and our DBO-CPD model. $x_{t}, r_{t}$, and $D_{t}$ represent a continuous variable of interest, the run length (hidden) variable, and documents, respectively. Our modeling contribution is to add texts $D_{1: t}$ for the accurate prediction of the run length $r_{t+1}$.

Schumaker and Chen, 2009; Gidófalvi and Elkan, 2001; Fung et al., 2003; Fung et al., 2002; Schumaker and Chen, 2006), tweets on Twitter ( $\mathrm{Si}$ et al., 2013; Wang et al., 2012; Bollen et al., 2011; St Louis et al., 2012), online chats (Kim et al., 2010; Gruhl et al., 2005), and blog posts (Peng et al., 2015; Mishne and Glance, 2006).

In experiments, we show that DBO-CPD can effectively distinguish whether an abrupt change is a change point or not in real-world datasets (see Section 3.1). Compared to previous BO-CPD models which explain the changes by human manual mappings, our DBO-CPD automatically explains the reasons why a change point has occurred by connecting the numerical sequence of data and textual features of news articles.

\section{Bayesian Online Change Point Detection}

This section will review our research problem, the change point detection (CPD) (Barry and Hartigan, 1993), and the Bayesian Online Change Point Detection (BO-CPD) (Adams and MacKay, 2007) and our model, Document Based Online Change Point Detection (DBO-CPD).
Let $x_{t} \in \mathbb{R}$ be a data observation at time $t$. We assume that a sequence of data $\left(x_{1}, x_{2}, \ldots, x_{t}\right)$ is composed of several non-overlapping productive partitions (Barry and Hartigan, 1992). The boundaries that separate the partitions is called the change points. Let $r$ be the random variable that denotes the run length, which is the number of time steps since the last change point was detected. $r_{t}$ is the current run at time $t . x_{t}^{\left(r_{t}\right)}$ denotes the most recent data corresponding to the run $r_{t}$.

\subsection{Online Recursive Detection}

To make an optimal prediction of the next data $x_{t+1}$, one may need to consider all possible run lengths $r_{t} \in \mathbb{N}$ and a probability distribution over run length $r_{t}$. Given a sequence of data up to time $t, x_{1: t}=\left(x_{1}, x_{2}, \ldots, x_{t}\right)$, the run length prediction problem is formalized as computing the joint probability of random variables $P\left(x_{t+1}, x_{1: t}\right)$. This distribution can be calculated in terms of the posterior distribution of run length at time $t, P\left(r_{t} \mid x_{1: t}\right)$, as follows:

$$
\begin{aligned}
P\left(x_{t+1}, x_{1: t}\right) & =\sum_{r_{t}} P\left(x_{t+1} \mid r_{t}, x_{t}^{\left(r_{t}\right)}\right) P\left(r_{t} \mid x_{1: t}\right) \\
& =\sum_{r_{t}} P\left(x_{t+1} \mid x_{t}^{\left(r_{t}\right)}\right) P\left(r_{t} \mid x_{1: t}\right) .(1)
\end{aligned}
$$

The predictive distribution $P\left(x_{t+1} \mid r_{t}, x_{t}^{\left(r_{t}\right)}\right)$ depends only on the most recent $r_{t}$ observations $x_{t}^{\left(r_{t}\right)}$. The posterior distribution of run length $P\left(r_{t} \mid x_{1: t}\right)$ can be computed recursively:

$$
P\left(r_{t} \mid x_{1: t}\right)=\frac{P\left(r_{t}, x_{1: t}\right)}{P\left(x_{1: t}\right)}
$$

where:

$$
P\left(x_{1: t}\right)=\sum_{r_{t}} P\left(r_{t}, x_{1: t}\right) .
$$

The joint distribution over run length $r_{t}$ and data $x_{1: t}$ can be derived by summing $P\left(r_{t}, r_{t-1}, x_{1: t}\right)$ over $r_{t-1}$ :

$$
\begin{aligned}
& P\left(r_{t}, x_{1: t}\right)=\sum_{r_{t-1}} P\left(r_{t}, r_{t-1}, x_{1: t}\right) \\
& =\sum_{r_{t-1}} P\left(r_{t}, x_{t} \mid r_{t-1}, x_{1: t-1}\right) P\left(r_{t-1}, x_{1: t-1}\right) \\
& =\sum_{r_{t-1}} P\left(r_{t} \mid r_{t-1}\right) P\left(x_{t} \mid r_{t-1}, x_{t}^{\left(r_{t}\right)}\right) P\left(r_{t-1}, x_{1: t-1}\right) .
\end{aligned}
$$

This formulation updates the posterior distribution of the run length given the prior over $r_{t}$ from $r_{t-1}$ and the predictive distribution of new data. 
However, the existing BO-CPD model (Adams and MacKay, 2007) specifies the conditional prior on the change point $P\left(r_{t} \mid r_{t-1}\right)$ in advance. This approach may lead to model biased predictions because the update formula highly relies on the predefined, fixed hazard rate $(h)$. Furthermore, BO$\mathrm{CPD}$ is incapable of incorporating external information that implicitly influences the observation and explains the reasons for the current change of the long-term trend.

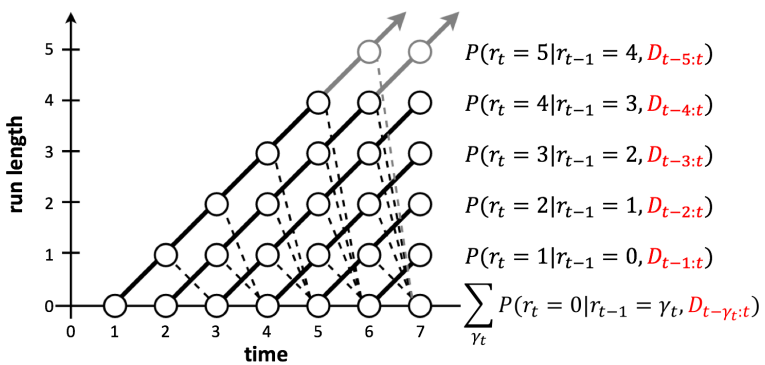

Figure 2: This figure illustrates the recursive updates of the posterior probability in the DBO-CPD model. Even the BO-CPD model only uses current and previous run length to calculate the posterior, DBO-CPD can utilize the series of text documents to compute the conditional probability accurately.

\subsection{Document-based Bayesian Online Change Point Detection}

This section explains our DBO-CPD model. To represent the text documents, we add a variable $\boldsymbol{D}$ which denotes a series of text documents related to the observed data as shown in Figure 1. Let $D_{t}$ be a set of $N_{t}$ text documents $D_{t}^{1}, D_{t}^{2}, \ldots, D_{t}^{N_{t}}$ that are indexed at time of publication $t$, where $N_{t}$ is the number of documents observed at time $t$. Then, we can rewrite the joint probability over the run length as:

$$
\begin{gathered}
P\left(r_{t}, x_{1: t}\right)=\sum_{r_{t-1}} \sum_{D_{t}^{\left(r_{t-1}\right)}} P\left(r_{t} \mid r_{t-1}, D_{t}^{\left(r_{t-1}\right)}\right) \\
P\left(x_{t} \mid r_{t-1}, x_{t}^{\left(r_{t-1}\right)}\right) P\left(r_{t-1}, x_{1: t-1}\right)
\end{gathered}
$$

where $D_{t}^{\left(r_{t}\right)}\left(=D_{t-r_{t}+1: t}\right)$ is the set of the $r_{t}$ most recent documents. Figure 2 illustrates the recursive updates of posterior probability where solid lines indicate that the probability mass is passed upwards and dotted lines indicate the probability that the current run length $r_{t}$ is set to zero.
Given documents $D_{t}^{\left(r_{t}\right)}$, the conditional probability is represented as follows:

$$
\begin{aligned}
& P\left(r_{t}=\gamma+1 \mid r_{t-1}=\gamma, D_{t}^{(\gamma)}\right) \\
= & \frac{P\left(r_{t-1}=\gamma, D_{t}^{(\gamma)} \mid r_{t}=\gamma+1\right) P\left(r_{t}=\gamma+1\right)}{\sum_{\bar{\gamma}=1}^{\gamma+1} P\left(r_{t-1}=\gamma, D_{t}^{(\gamma)} \mid r_{t}=\bar{\gamma}\right) P\left(r_{t}=\bar{\gamma}\right)} \\
= & \frac{P\left(r_{t-1}=\gamma, D_{t}^{(\gamma)} \mid r_{t}=\gamma+1\right) P_{g a p}(\gamma+1)}{\sum_{\bar{\gamma}=1}^{\gamma+1} P\left(r_{t-1}=\gamma, D_{t}^{(\gamma)} \mid r_{t}=\bar{\gamma}\right) P_{g a p}(\bar{\gamma})}
\end{aligned}
$$

where $P_{g a p}$ is the distribution of intervals between consecutive change-points. As the BO-CPD model (Adams and MacKay, 2007), we assume the simplest case where the probability of a changepoint at every step is constant if the length of a segment is modeled by a discrete exponential (geometric) distribution as:

$$
P_{g a p}\left(r_{t} \mid \lambda\right)=\lambda \exp ^{-\lambda r_{t}}
$$

where $\lambda>0$, a rate parameter, is the parameter of the distribution.

The update rule for the prior distribution on $r_{t}$ makes the computation of the joint distribution tractable, $\sum_{\bar{\gamma}=1}^{\gamma+1} P\left(r_{t-1}=\gamma, D_{t}^{(\gamma)} \mid r_{t}=\bar{\gamma}\right) \cdot P_{\text {gap }}(\bar{\gamma})$. Because $r_{t}$ can only be increased to $\gamma+1$ or set to 0 , the conditional probability is as follows:

$$
\begin{aligned}
& P\left(r_{t}=\gamma+1 \mid r_{t-1}=\gamma, D_{t}^{(\gamma)}\right) \\
& =\frac{T_{D}(t, \gamma \mid \gamma+1)}{T_{D}(t, \gamma \mid \gamma+1)+T_{D}(t, \gamma \mid 0)}
\end{aligned}
$$

where the function $T_{D}(t, \alpha \mid \bar{\alpha})$ is an abbreviation of $P\left(r_{t-1}=\alpha, D_{t}^{(\alpha)} \mid r_{t}=\bar{\alpha}\right)$. In Equation (6), $T_{D}(t, \gamma \mid \gamma+1)=P\left(r_{t-1}=\gamma, D_{t}^{(\gamma)} \mid r_{t}=\gamma+1\right)$ is the joint probability of the run length $r_{t-1}$ and a set of documents $D_{t}^{(\gamma)}$ when no change has occurred at time $t$ and the run length becomes $\gamma+1$. Therefore, we can simplify the equation by removing $r_{t-1}=\gamma$ from the condition as follows:

$$
T_{D}(t, \gamma \mid \gamma+1)=P\left(D_{t}^{(\gamma)} \mid r_{t}=\gamma+1\right) .
$$

We represent the distribution of words by the bagof-words model. Let $D_{t}^{i}$ be the set of $M$ words that is part of the $i$ th document at time $t$, i.e. $D_{t}^{i}=\left\{d_{t}^{i, 1}, d_{t}^{i, 2}, \ldots, d_{t}^{i, M}\right\}$. In the model, we assume that the probability of word $d_{t}^{i, j}$ is independent and identically distributed (iid) given a run 
length parameter $r_{t}$. In this setting, the conditional probability of the words takes the following form:

$P\left(D_{t}^{(\gamma)} \mid r_{t}=\gamma+1\right)=\frac{1}{Z} \prod_{i, j} P\left(d_{t}^{i, j} \mid r_{t}=\gamma+1\right)$.

The conditional probability $P\left(d_{t}^{i, j} \mid r_{t}=\gamma+1\right)$ is represented by two generative models, $\phi_{\mathbf{w f}}$ and $\phi_{\text {wi }}$ which illustrates word frequency and word impact, respectively. The key intuition of word frequency is that a word tends to close to a change point if a word has been frequently seen in articles, published when there was a rapid change. The key intuition of word impact is that how much does a word lose information in time which will be discussed in next section. In our paper, we use the unnormalized beta distribution of the weights of words to represent the exponential decays. The probability $P\left(D_{t}^{(\gamma)} \mid r_{t}=\gamma+1\right)$ can be represented recursively as:

$$
\begin{aligned}
P & \left(D_{t}^{(\gamma)} \mid r_{t}=\gamma+1\right)=P\left(D_{t}^{(\gamma)} \mid \gamma+1\right) \\
\propto & \phi_{\mathbf{w i}}\left(D_{t}^{(\gamma)} \mid \gamma+1\right) \cdot \phi_{\mathbf{w r}}\left(D_{t}^{(\gamma)} \mid \gamma+1\right) \\
= & \phi_{\mathbf{w i}}\left(D_{t} \mid \gamma+1\right) \cdot \phi_{\mathbf{w f}}\left(D_{t} \mid \gamma+1\right) \\
& \quad \phi_{\mathbf{w i}}\left(D_{t-1}^{(\gamma-1)} \mid r_{t-1}=\gamma\right) \cdot \phi_{\mathbf{w f}}\left(D_{t-1}^{(\gamma-1)} \mid r_{t-1}=\gamma\right) \\
= & \prod_{i, j} \phi_{\mathbf{w i}}\left(d_{t}^{i, j} \mid \gamma+1\right) \cdot \phi_{\mathbf{w f}}\left(d_{t}^{i, j} \mid \gamma+1\right) \\
& \quad \phi_{\mathbf{w i}}\left(D_{t-1}^{(\gamma-1)} \mid r_{t-1}=\gamma\right) \cdot \phi_{\mathbf{w f}}\left(D_{t-1}^{(\gamma-1)} \mid r_{t-1}=\gamma\right)
\end{aligned}
$$

where:

$$
\phi_{\mathbf{w f}}\left(d_{t}^{x, y} \mid \gamma\right)=\frac{\operatorname{count}\left(d_{t}^{x, y}, r_{t}=\gamma\right)}{\sum_{i, j} \operatorname{count}\left(d_{t}^{i, j}, r_{t}=\gamma\right)} .
$$

Here, $\phi_{\mathbf{w i}}\left(d_{t}^{x, y} \mid \gamma\right)$ and $\phi_{\mathbf{w f}}\left(d_{t}^{x, y} \mid \gamma\right)$ are empirical potentials which contribute to represent $P\left(d_{t}^{i, j} \mid \gamma\right)$. $\phi_{\mathbf{w i}}(\cdot)$ is explained in Section 2.3. Here, $\operatorname{count}(E)$ is the number of times event $E$ appears in the dataset. In Equation (9), $\tau_{t}$ is the time gap (difference) between $t$ and the time when a document is generated, and $d^{i, j}$ represents a document without considering the time domain.

$T_{D}(t, \gamma \mid 0)$ is represented as follows:

$$
\begin{aligned}
& P\left(r_{t-1}=\gamma, D_{t}^{(\gamma)} \mid r_{t}=0\right) \\
& =P\left(r_{t-1}=\gamma \mid r_{t}=0\right) P\left(D_{t}^{(\gamma)} \mid r_{t}=0\right) \\
& =H(\gamma+1) P\left(D_{t}^{(\gamma)} \mid r_{t}=0\right)
\end{aligned}
$$

where $H(\tau)$ is the hazard function (Forbes et al., 2011),

$$
H(\tau)=\frac{P_{g a p}(\tau)}{\sum_{t=\tau}^{\infty} P_{g a p}(t)} .
$$

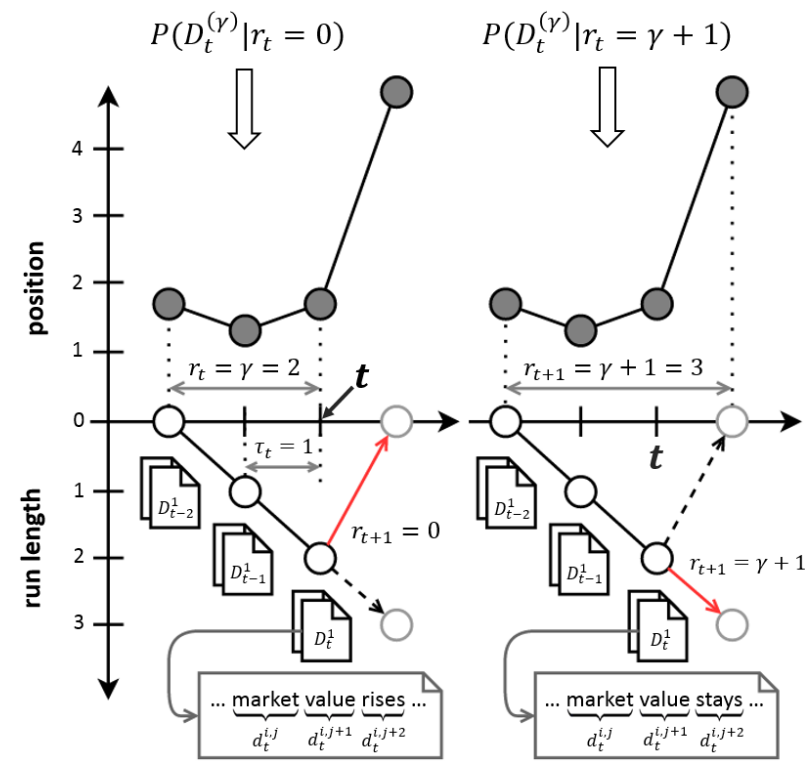

Figure 3: This figure illustrates how our Equation (9) is calculated and how it determines whether a change occurs or not. If the same data is given, BO-CPD gives us the same answer to a question whether an abrupt change at time $t$ is a change point or not. However, DBO-CPD uses documents $D_{t}^{\gamma}$ for its prediction to incorporate the external information which cannot be inferred only from the data.

When $P_{g a p}$ is the discrete exponential distribution, the hazard function is constant at $H(\tau)=1 / \lambda$ (Adams and MacKay, 2007).

As an illustrative example, suppose that we found a rapid change in Google stock three days ago. Today at $t=3$, we want to know how the articles are written and whether it will affect the change tomorrow $(t=4)$. As shown in Figure 3, we can calculate what degree a word, for example rises or stays, is likely to appear in articles published since today, which is $P\left(D_{t}^{(\gamma)} \mid r_{t}=\gamma+1\right)$, and this probability leads us to predict run lengths from the texts. Documents for each $\tau_{t}=0,1$ and 2 are generated from the generative models with a given predicted run length through recursive calculation of the Bayesian models which enables online prediction as shown in Equation (9). This is the main contribution of this paper that enables DBO-CPD to infer change points accurately with information included in text documents. 


\subsection{Generative Models Trained from Regression}

Let $D \in \mathbb{R}^{T \times N \times M}$ be $N$ documents of news articles which consist of $M$ vocabulary over time domain $T . D_{t}^{i} \in \mathbb{R}^{M}$ is the $i$ th document of a set of documents generated at time $t$, and define $r \in \mathbb{R}^{N}$ as the corresponding set of the run length, which is a time gap between the time when the document is generated and the next change point occurs. Then, given a text document $D_{t}^{i}$, we seek to predict the value of run length $r$ by learning a parameterized function $f$ :

$$
\hat{r}=f\left(D_{t}^{i} ; \mathbf{w}\right)
$$

where $\mathbf{w} \in \mathbb{R}^{d}$ are the weights of text features for $d_{t}^{i, 1}, d_{t}^{i, 2}, \ldots, d_{t}^{i, M}$ which compose documents $D_{t}^{i}$. From a collection of $N$ documents, we use linear regression which is trained by solving the following optimization problem:

$$
\min _{\mathbf{w}, D_{t}^{i}} f\left(D_{t}^{i} ; \mathbf{w}\right) \equiv \mathbf{C} \sum_{\mathbf{i}=\mathbf{1}}^{\mathbf{N}} \xi\left(\mathbf{w}, \mathbf{D}_{\mathbf{t}}^{\mathbf{i}}, \mathbf{r}_{\mathbf{t}}\right)+\mathbf{r}(\mathbf{w})
$$

where $r(\mathbf{w})$ is the regularization term and $\xi\left(\mathbf{w}, \mathbf{D}_{\mathbf{t}}^{\mathbf{i}}, \mathbf{r}_{\mathbf{t}}\right)$ is the loss function. Parameter $C>$ 0 is a user-specified constant for balancing $r(\mathbf{w})$ and the sum of losses.

Let $h$ be a function from a document into a vector-space representation $\in \mathbb{R}^{d}$. In linear regression, the function $f$ takes the form:

$$
f\left(D_{t}^{i} ; \mathbf{w}\right)=h\left(D_{t}^{i}\right)^{\top} \mathbf{w}+\epsilon
$$

where $\epsilon$ is Gaussian noise.

Figure 4 illustrates how we trained a linear regression model on a sample article. One issue is that the run length can not be trained directly. Suppose that we train $r_{5}=0$ into regression, the weight $\mathbf{w}$ of the model will become 0 even though the set of words contained in $D_{5}^{j}, \forall j \in\{1, \ldots, T\}$ is composed of salient words which can incur a possible future change point. To solve this interpretability problem, we trained the weight in the inverse exponential domain for the predicted variable, predicting $e^{-r_{t}}$ instead of $r_{t}$. In this setting, the predicted run-length takes the form:

$$
e^{-\hat{r_{t}}}=h\left(D_{t}\right)^{\top} \mathbf{w}+\epsilon .
$$

By this method, the regression model can give a high weight to a word which often appears close to change points. We can interpret that highly

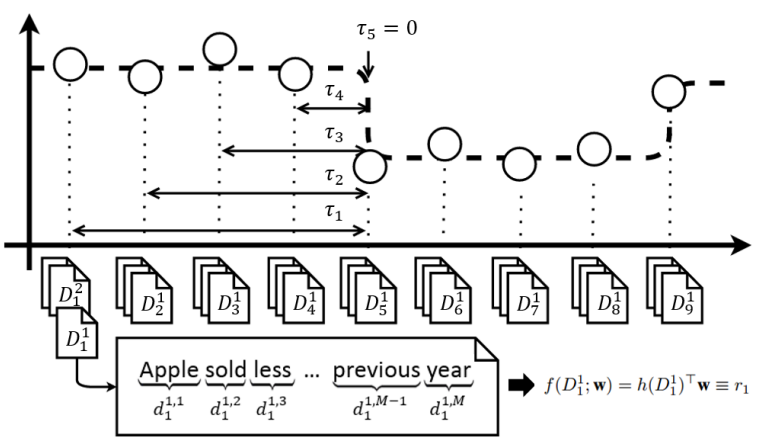

Figure 4: This figure illustrates a graphical representation of how we train a generative model from a regression problem. We use a regression model to predict time gap $r_{t}$ between the release date of article and the nearest future change point. The weights of regression model are changed into the negative exponential scale to be considered as word impact.

weighted words $d$ are more closely related to an outbreak of changes than lower weighted words.

With $\mathbf{w}$, we can rewrite the probability of $d, \tau_{t}$ given $\mathbf{w}$ as:

$$
\begin{aligned}
\phi_{\mathbf{w i}}\left(d, \tau_{t}\right) & \propto \mathbf{w}_{d} \cdot\left(\exp \left(-1 / \mathbf{w}_{d}\right)\right)^{\tau_{t}} \\
& =\mathbf{w}_{d} \cdot \exp \left(-\tau_{t} / \mathbf{w}_{d}\right) .
\end{aligned}
$$

The potential, $\phi_{\text {wi }}$, can also be represented recursively as follows:

$$
\phi_{\mathbf{w i}}\left(d, \tau_{t+1}\right)=\phi_{\mathbf{w i}}\left(d, \tau_{t}\right) \cdot \exp \left(-1 / \mathbf{w}_{d}\right),
$$

since given a word $d, \tau_{t+1}=\tau_{t}+1$ holds.

\section{Experiments}

Now we explain experiments of DBO-CPD in two real-world datasets, stock prices and movie revenues. The first case is the historical end-of-day stock prices of five information technology corporations. In the second dataset, we examine daily film revenues averaged by the number of theaters.

\subsection{Datasets}

In the stock price dataset, we gather data for five different companies: Apple (AAPL), Google (GOOG), IBM (IBM), Microsoft (MSFT), and Facebook (FB). These companies were selected because they were the top 5 ranked in market value in 2015.

We chose these technology companies because the announcement of new IT products and features and the interests of public media tend to be higher 

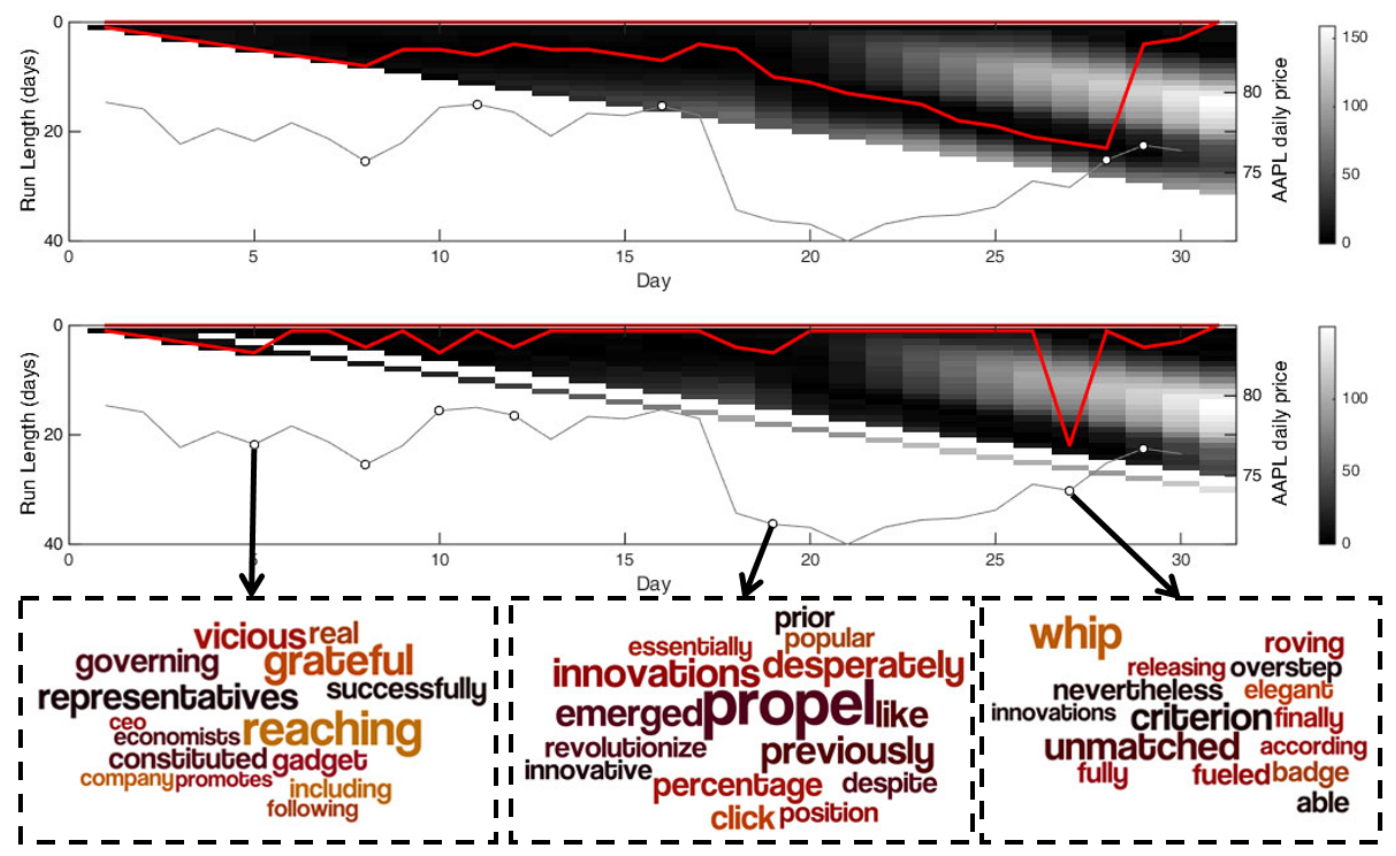

Figure 5: (a) Two plots show the results of BO-CPD (top) and DBO-CPD (middle) on Apple stock prices in January 2014. The stock price is plotted in light gray, with the predictive change points drawn as small circles. The red line represents the most likely predicted run-lengths for each day. The bottom figures are a set of visualizations of the top 15 strongly weighted words which are found at selected change points which BO-CPD is unable to predict. The size of each word represents the weight of its textual features learned during the training of the regression model.

and lead to many news articles. We use the historical stock price data from the Google Finance service. ${ }^{1}$.

\begin{tabular}{|l||r|r|r|}
\hline category & words & documents & words/doc \\
\hline AAPL & $15.0 \mathrm{M}$ & 29,459 & 509 \\
AAPL:N & $11.0 \mathrm{M}$ & 18,896 & 581 \\
GOOG & $15.0 \mathrm{M}$ & 29,422 & 511 \\
GOOG:N & $8.2 \mathrm{M}$ & 13,658 & 603 \\
IBM & $26.7 \mathrm{M}$ & 45,741 & 583 \\
IBM:N & $3.4 \mathrm{M}$ & 4,741 & 726 \\
MSFT & $20.5 \mathrm{M}$ & 35,905 & 570 \\
MSFT:N & $3.5 \mathrm{M}$ & 5,070 & 681 \\
FB & $18.9 \mathrm{M}$ & 38,168 & 495 \\
FB:N & $4.3 \mathrm{M}$ & 6,625 & 645 \\
\hline KNGHT & $14.4 \mathrm{M}$ & 16,874 & 852 \\
INCPT & $12.1 \mathrm{M}$ & 17,155 & 705 \\
AVGR & $3.5 \mathrm{M}$ & 6,476 & 537 \\
FRZ & $6.8 \mathrm{M}$ & 15,021 & 454 \\
INTRS & $4.2 \mathrm{M}$ & 7,846 & 538 \\
\hline
\end{tabular}

Table 1: Dimensions of the datasets used in this paper, after tokenizing and filtering the news articles. ' $: \mathbf{N}$ ' means the articles are collected with additional 'NASDAQ:' search query.

The second dataset is a set of movie revenues averaged by the number of theaters for five months from the release date of film. We target 5 different

\footnotetext{
${ }^{1}$ https://www.google.com/finance
}

movies: The Dark Knight (KNGHT), Inception (INCPT), The Avengers (AVGR), Frozen (FRZ) and Interstellar (INTRS), because these movies are on highest-grossing movie list and also are screened recently. The cumulative daily revenue per theater is collected from 'Box Office Mojo' (www.boxofficemojo.com).

News articles are collected from Google News and we use Google search queries to extract specific articles related to each dataset in a specific time period. During the online article crawling, we store not only the titles of articles, HTML documents, and publication dates, but also the number of related articles. The number of articles is used to differentiate the weight of news articles during the training of regression. In the case of stock price data, we use two different queries to decrease noise. First, we search with the company name such as 'Google'. Then, we use queries specific to stock 'NASDAQ:' to make the content of articles to be highly relevant to the stock market. In case of movie data, we search with the movie title with the additional word 'movie' to only collect articles related to the target movie.

With these collected articles, we used two ar- 
ticle extractors, newspaper (Ou-Yang, 2013) and python-goose (Grangier, 2013), to automate the text extraction of 291,057 HTML documents. After preprocessing, we could successfully extract texts from 287,389 (98.74\%) HTMLs.

\subsection{Textual Feature Representation}

After extracting texts from HTMLs, we tokenize the texts into words. We use three different tokenization methods which are downcasing the characters, punctuation removal, and removing English stop words. Table 1 shows the statistics on the corpora of collected news articles.

With these article corpora, we use a bag-ofwords $(\mathrm{BoW})$ representation to change each word into a vector representation where words from articles are indexed and then weighted. Using these vectors, we adopt three document representations, TF, TFIDF, and LOG1P, which extend BoW representation. TF and TFIDF (Sparck Jones, 1972) calculate the importance of a word to a set of documents based on term frequency. LOG1P (Kogan et al., 2009) calculates the logarithm of the word frequencies.

\subsection{Training BO-CPD}

As we noted earlier, we use BO-CPD to train the regression model to learn high weight for words which are more related to changes. When we choose the parameters for the Gaussian Process of BO-CPD, we try to find the value which makes the distance of intervals between predicted change points around 1-2 weeks. This is because we assume that the information included in the articles will have an immediate effect on the data right after it is published to the public, so the external information in texts will indicate the short-term causes for a future change.

For the reasonable comparison of BO-CPD and DBO-CPD, we use the same parameter for the Gaussian Process in both models. After several experiments we found that $a=1$ and $b=1$ for the Gaussian Process and $\lambda_{\text {gap }}=250$ is appropriate to train BO-CPD in the stock and film datasets. We separate the training and testing examples for cross-validation at a ratio of $2: 1$ for each year. Then we train each model differently by year.

\subsection{Learning the strength parameter $w$ from Regression}

The weight $\mathbf{w}$ of the regression model gives us an intuition of how a word is important which affect

\begin{tabular}{|l||l|l|l|l|l|}
\hline & 2010 & 2011 & 2012 & 2013 & 2014 \\
\hline AAPL BO-CPD & 14.93 & 16.33 & 16.24 & 14.44 & 17.63 \\
\hline AAPL DBO-CPD I & $\mathbf{1 4 . 8 1}$ & 16.22 & 16.20 & $\mathbf{1 4 . 2 1}$ & 17.12 \\
\hline AAPL DBO-CPD II & 15.15 & $\mathbf{1 6 . 2 0}$ & $\mathbf{1 6 . 1 4}$ & 14.40 & $\mathbf{1 7 . 1 1}$ \\
\hline GOOG BO-CPD & $\mathbf{1 5 . 0 3}$ & 15.65 & 15.49 & 19.43 & 19.04 \\
\hline GOOG DBO-CPD I & 15.48 & 15.92 & $\mathbf{1 5 . 2 1}$ & 19.24 & 19.07 \\
\hdashline GOOG DBO-CPD II & 15.31 & $\mathbf{1 5 . 6 2}$ & 15.36 & $\mathbf{1 9 . 2 0}$ & $\mathbf{1 9 . 0 2}$ \\
\hline IBM BO-CPD & 17.10 & 17.83 & 17.42 & 16.25 & 16.30 \\
\hline IBM DBO-CPD I & 17.66 & $\mathbf{1 7 . 8 1}$ & 17.40 & 16.20 & $\mathbf{1 6 . 0 4}$ \\
\hline IBM DBO-CPD II & $\mathbf{1 7 . 0 4}$ & 17.82 & $\mathbf{1 7 . 3 8}$ & $\mathbf{1 6 . 1 4}$ & 16.39 \\
\hline MSFT BO-CPD & 12.41 & 11.91 & 14.51 & 15.60 & 17.25 \\
\hline MSFT DBO-CPD I & 12.33 & 12.60 & 14.48 & $\mathbf{1 4 . 9 2}$ & $\mathbf{1 6 . 4 3}$ \\
\hline MSFT DBO-CPD II & $\mathbf{1 2 . 2 1}$ & $\mathbf{1 1 . 7 9}$ & $\mathbf{1 4 . 4 6}$ & 15.00 & 16.46 \\
\hline FB BO-CPD & N/A & N/A & $\mathbf{1 2 . 3 2}$ & 13.07 & 16.68 \\
\hdashline FB DBO-CPD I & N/A & N/A & 12.34 & 13.00 & 16.24 \\
\hline FB DBO-CPD II & N/A & N/A & 12.43 & $\mathbf{1 2 . 9 8}$ & $\mathbf{1 6 . 2 5}$ \\
\hline
\end{tabular}

Table 2: Negative log likelihood of five stocks (Apple, Google, IBM, Microsoft, and Facebook) without and with our model per year from 2010 to 2014. DBO-CPD I represents the experiments without 'NASDAQ:' as a search query and DBOCPD II is the result of articles searched with 'NASDAQ:'. Facebook data is not available before the year 2012 .

to the length of the current run. With the predicted run length calculated in Section 3.3, we change the run length domain $r \in \mathbb{R}$ into $0 \leq r \leq 1$ by predicting $e^{r_{t}}$ rather than $r_{t}$ to solve the interpretability problem. Therefore, we can think of a high weight $w_{i}$ as a powerful word which changes the current run length $r$ to 0 . To maintain the scalability of $\mathbf{w}$, we normalize the weight by rescaling the range into $\mathbf{w} \in[-1,1]$. With the word representation calculated in Section 3.2, we train the regression model by using the number of relevant articles as the importance weight of training.

\subsection{Results}

We evaluate the performance of BO-CPD and DBO-CPD by comparing the negative log likelihood (NLL) (Turner et al., 2009) of two models at time $t$ as:

$$
\log p\left(x_{1: T} \mid \mathbf{w}\right)=\sum_{t=1}^{T} \log p\left(x_{t} \mid x_{1: t-1}, \mathbf{w}\right) .
$$

We calculate the marginal NLL by year and the results are described in Table 2 and Table 3. (Facebook data is not available before the year 2012.) The difference between DBO-CPD I and DBOCPD II is whether the search queries include 'NASDAQ'. In stock data sets of 5 years, our model outperforms BO-CPD in Apple, Google, IBM, Microsoft dataset. The improvements of 


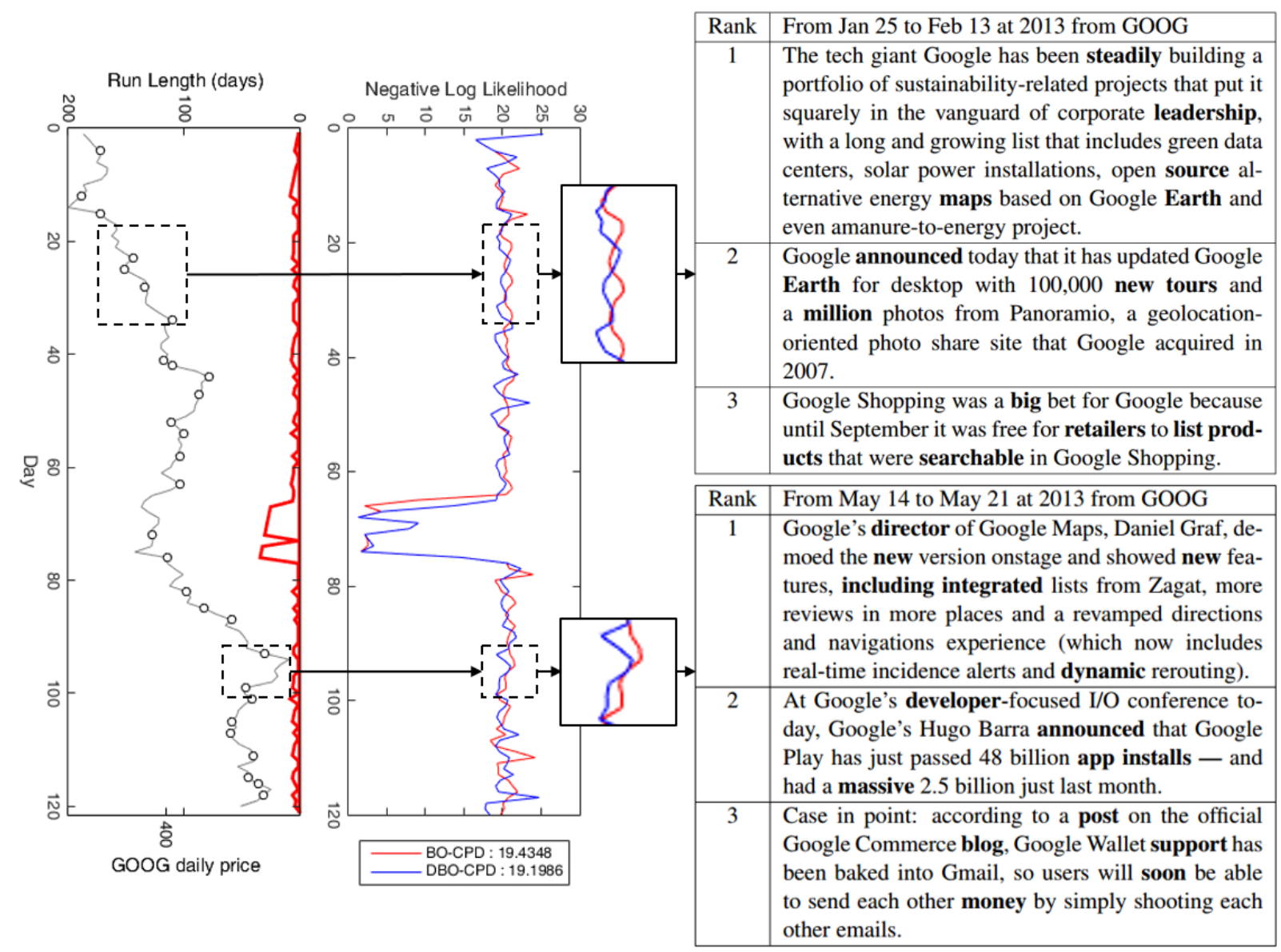

Figure 6: (b) The left plot illustrates daily stock prices of Google in 2013 from early January to late May. The black line represents the stock price, black circles indicate the predicted change points, and the red line shows the predicted run length calculated by DBO-CPD. The middle plot shows the negative log likelihood (NLL) of BO-CPD and DBO-CPD on the same data. The overall marginal NLL of DBO-CPD (19.1964) is smaller than BO-CPD (19.3438). The two zoomed intervals are the two longest intervals where the negative log likelihood of DBO-CPD is smaller than BO-CPD. The right table shows the sentences whose run length predicted by the regression model (described in Section 2.3) are the highest at the two zoomed points, which means the sentences are likely to appear near feature change points. The boldface words are the top 5 most strongly-weighted terms in the regression model.

DBO-CPD compared to the BO-CPD is statistically significant with $90 \%$ confidence in the four stocks except for stock of Facebook. We also found that most of the DBO-CPD II shows better results than DBO-CPD I and BO-CPD in most datasets due to noise reduction of texts through the additional search query 'NASDAQ:'. Out of 23 datasets, APPL in 2010 and FB in 2012 are the only datasets where NLLs of BO-CPD is smaller (better) than NLLs of DBO-CPD.

One of the advantages of using a linear model is that we can investigate what the model discovers about different terms. As shown in Figure 5, we can find negative semantic words such as vicious, whip, and desperately, and words represent- ing the status of a company like propel, innovations, and grateful are the most strongly-weighted terms in the regression model. We analyze and visualize some change points where NLL of DBOCPD is lower than NLL of BO-CPD. The results are shown in Figure 6 and three sentences are the top 3 most weighted sentences in the regression model for two changes with the boldface words of top 5 strongly weighted terms like the terms big, money, and steadily. A particularly interesting case is the term earth which is found between Jan. 25 and Feb. 13 in 2013. After we investigated articles where the sentence is included, we found that Google announced a new tour guide feature in Google Earth on Jan. 31 and after this announce- 


\begin{tabular}{|l||c|}
\hline \multicolumn{1}{|c||}{} & NLL \\
\hline KNGHT BO-CPD & 39.76 \\
\hline KNGHT DBO-CPD I & $\mathbf{3 9 . 5 4}$ \\
\hline INCPT BO-CPD & 55.60 \\
\hline INCPT DBO-CPD I & $\mathbf{5 5 . 5 4}$ \\
\hline AVGR BO-CPD & 32.12 \\
\hline AVGR DBO-CPD I & $\mathbf{3 2 . 1 0}$ \\
\hline FRZ BO-CPD & 51.25 \\
\hline FRZ DBO-CPD I & $\mathbf{5 1 . 0 4}$ \\
\hline INT BO-CPD & 38.49 \\
\hline INT DBO-CPD I & $\mathbf{3 8 . 3 1}$ \\
\hline
\end{tabular}

Table 3: Negative log likelihood (NLL) of five movies (The Dark Knight, Inception, Avengers, Frozen, and Interstellar) without and with our model for 1 year from the release date of each movie.

ment the stock price increased. We can also find that the word million is also a positive term which can predict a new change in the near feature.

\section{Conclusions}

In this paper, we propose a novel generative model for online inference to find change points from non-stationary time-series data. Unlike previous approaches, our model can incorporate external information in texts which may includes the causes of signal changes. The main contribution of this paper is to combine the generative model for online change points detection and a regression model learned from the weights of words in documents. Thus, our model accurately infers the conditional prior of the change points and automatically explains the reasons of a change by connecting the numerical sequence of data and textual features of news articles.

\section{Future work}

Our DBO-CPD can be improved further by incorporating more external information beyond documents. In principle, our DBO-CPD can incorporate other features if they are vectorized into a matrix form. Our implementation currently only uses the simple bag of words models (TF, TFIDF and LOG1P) to improve the baseline GP-based CPD models by bringing documents into change point detection. One possible direction of future work would explore ways to fully represent the rich information in texts by extending the text features and language representations like continuous bagof-words (CBOW) models (Mikolov et al., 2013) or Global vectors for word representation (GloVe) (Pennington et al., 2014).

\section{Acknowledgments}

This work was supported by Basic Science Research Program through the National Research Foundation of Korea (NRF) grant funded by the Ministry of Science, ICT \& Future Planning (MSIP) (NRF- 2014R1A1A1002662), the NRF grant funded by the MSIP (NRF2014M2A8A2074096).

\section{References}

Ryan Prescott Adams and David JC MacKay. 2007. Bayesian online changepoint detection. arXiv preprint arXiv:0710.3742.

Daniel Barry and John A Hartigan. 1992. Product partition models for change point problems. The Annals of Statistics, pages 260-279.

Daniel Barry and John A Hartigan. 1993. A bayesian analysis for change point problems. Journal of the American Statistical Association, 88(421):309-319.

Johan Bollen, Huina Mao, and Xiaojun Zeng. 2011. Twitter mood predicts the stock market. Journal of Computational Science, 2(1):1-8.

Jie Chen and AK Gupta. 1997. Testing and locating variance changepoints with application to stock prices. Journal of the American Statistical Association, 92(438):739-747.

Siddhartha Chib. 1998. Estimation and comparison of multiple change-point models. Journal of econometrics, 86(2):221-241.

Pao-Shin Chu and Xin Zhao. 2004. Bayesian changepoint analysis of tropical cyclone activity: The central north pacific case. Journal of Climate, 17(24):4893-4901.

Catherine Forbes, Merran Evans, Nicholas Hastings, and Brian Peacock. 2011. Statistical distributions. John Wiley \& Sons.

Gabriel Pui Cheong Fung, Jeffrey $\mathrm{Xu} \mathrm{Yu}$, and Wai Lam. 2002. News sensitive stock trend prediction. In Advances in knowledge discovery and data mining, pages 481-493. Springer.

Gabriel Pui Cheong Fung, Jeffrey $\mathrm{Xu}$ Yu, and Wai Lam. 2003. Stock prediction: Integrating text mining approach using real-time news. In IEEE International Conference on Computational Intelligence for Financial Engineering, pages 395-402.

Gyozo Gidófalvi and Charles Elkan. 2001. Using news articles to predict stock price movements. Department of Computer Science and Engineering, University of California, San Diego.

Xavier Grangier. 2013. Python-goose - article extractor. 
Daniel Gruhl, Ramanathan Guha, Ravi Kumar, Jasmine Novak, and Andrew Tomkins. 2005. The predictive power of online chatter. In Proceedings of the Eleventh ACM SIGKDD International Conference on Knowledge Discovery and Data Mining, pages 78-87.

William Gu, Jaesik Choi, Ming Gu, Horst Simon, and Kesheng Wu. 2013. Fast change point detection for electricity market analysis. In IEEE International Conference on Big Data, pages 50-57.

Der-Ann Hsu. 1977. Tests for variance shift at an unknown time point. Applied Statistics, pages 279 284.

Su Nam Kim, Lawrence Cavedon, and Timothy Baldwin. 2010. Classifying dialogue acts in one-onone live chats. In Proceedings of the 2010 Conference on Empirical Methods in Natural Language Processing, pages 862-871.

Shimon Kogan, Dimitry Levin, Bryan R Routledge, Jacob S Sagi, and Noah A Smith. 2009. Predicting risk from financial reports with regression. In Proceedings of Human Language Technologies: Conference of the North American Chapter of the Association for Computational Linguistics, pages 272280.

Gary Koop and Simon M. Potter. 2007. Estimation and forecasting in models with multiple breaks. The Review of Economic Studies, 74(3):pp. 763-789.

Tomas Mikolov, Kai Chen, Greg Corrado, and Jeffrey Dean. 2013. Efficient estimation of word representations in vector space. arXiv preprint arXiv:1301.3781.

Gilad Mishne and Natalie S Glance. 2006. Predicting movie sales from blogger sentiment. In AAAI Spring Symposium: Computational Approaches to Analyzing Weblogs, pages 155-158.

Joel Nothman, Matthew Honnibal, Ben Hachey, and James R Curran. 2012. Event linking: Grounding event reference in a news archive. In Proceedings of the 50th Annual Meeting of the Association for Computational Linguistics (ACL), pages 228-232.

Michael Osborne. 2010. Bayesian Gaussian processes for sequential prediction, optimisation and quadrature. Ph.D. thesis, Oxford University New College.

Lucas Ou-Yang. 2013. newspaper - news, full-text, and article metadata extraction.

Baolin Peng, Jing Li, Junwen Chen, Xu Han, Ruifeng $\mathrm{Xu}$, and Kam-Fai Wong. 2015. Trending sentimenttopic detection on twitter. In Computational Linguistics and Intelligent Text Processing, pages 6677. Springer.

Jeffrey Pennington, Richard Socher, and Christopher D Manning. 2014. Glove: Global vectors for word representation. Proceedings of the Empiricial
Methods in Natural Language Processing (EMNLP 2014), 12:1532-1543.

Robert Schumaker and Hsinchun Chen. 2006. Textual analysis of stock market prediction using financial news articles. AMCIS 2006 Proceedings, page 185.

Robert P Schumaker and Hsinchun Chen. 2009. Textual analysis of stock market prediction using breaking financial news: The azfin text system. ACM Transactions on Information Systems (TOIS), 27(2):12.

Jianfeng Si, Arjun Mukherjee, Bing Liu, Qing Li, Huayi Li, and Xiaotie Deng. 2013. Exploiting topic based twitter sentiment for stock prediction.

AFM Smith. 1975. A bayesian approach to inference about a change-point in a sequence of random variables. Biometrika, 62(2):407-416.

Karen Sparck Jones. 1972. A statistical interpretation of term specificity and its application in retrieval. Journal of documentation, 28(1):11-21.

Connie St Louis, Gozde Zorlu, et al. 2012. Can twitter predict disease outbreaks? BMJ, 344 .

DA Stephens. 1994. Bayesian retrospective multiplechangepoint identification. Applied Statistics, pages 159-178.

Mark Steyvers and Scott Brown. 2005. Prediction and change detection. In Advances in Neural Information Processing Systems (NIPS), pages 1281-1288.

Ryan Turner, Yunus Saatci, and Carl Edward Rasmussen. 2009. Adaptive sequential bayesian change point detection.

Udo von Toussaint. 2011. Bayesian inference in physics. Reviews of Modern Physics, 83(3):943.

Hao Wang, Dogan Can, Abe Kazemzadeh, François Bar, and Shrikanth Narayanan. 2012. A system for real-time twitter sentiment analysis of 2012 us presidential election cycle. In Proceedings of the ACL 2012 System Demonstrations, pages 115-120. Association for Computational Linguistics.

Xiang Xuan and Kevin Murphy. 2007. Modeling changing dependency structure in multivariate time series. In Proceedings of the 24th International Conference on Machine Learning (ICML), pages 1055-1062.

Xin Zhao and Pao-Shin Chu. 2010. Bayesian changepoint analysis for extreme events (typhoons, heavy rainfall, and heat waves): An rjmcmc approach. Journal of Climate, 23(5):1034-1046. 